University of New Orleans

ScholarWorks@UNO

2008

\title{
High-frequency characterization of Permalloy nanosized strips using network analyzer ferromagnetic resonance
}

Bijoy K. Kuanr

Radek Lopusnik

Leszek M. Malkinski

University of New Orleans

Matt Wenger

Minghui Yu

University of New Orleans

See next page for additional authors

Follow this and additional works at: https://scholarworks.uno.edu/phys_facpubs

Part of the Physics Commons

\section{Recommended Citation}

J. Appl. Phys. 103, 07C508 (2008)

This Article is brought to you for free and open access by the Department of Physics at ScholarWorks@UNO. It has been accepted for inclusion in Physics Faculty Publications by an authorized administrator of ScholarWorks@UNO.

For more information, please contact scholarworks@uno.edu. 


\section{Authors}

Bijoy K. Kuanr, Radek Lopusnik, Leszek M. Malkinski, Matt Wenger, Minghui Yu, Donald Scherer II, Z Celinski, and R E. Camley 


\title{
High-frequency characterization of Permalloy nanosized strips using network analyzer ferromagnetic resonance
}

\author{
Bijoy K. Kuanr, ${ }^{1, a)}$ Radek Lopusnik, ${ }^{2}$ Leszek M. Malkinski, ${ }^{3}$ Matt Wenger, ${ }^{1}$ Minghui $Y u,{ }^{3}$ \\ Donald Scherer II, ${ }^{3}$ R. E. Camley, ${ }^{1}$ and Z. Celinski ${ }^{1}$ \\ ${ }^{1}$ Department of Physics, University of Colorado, Colorado Springs, Colorado 80918, USA \\ ${ }^{2}$ Seagate Technology, Bloomington, Minnesota 55435, USA \\ ${ }^{3}$ Advanced Materials Research Institute, University of New Orleans, New Orleans, Louisiana 70148, USA
}

(Presented on 6 November 2007; received 27 September 2007; accepted 21 December 2007; published online 20 February 2008)

\begin{abstract}
We report on the dynamic properties of Permalloy nanostrips at gagahertz frequencies. The thickness of the strips is $100 \mathrm{~nm}$, strip width is $300 \mathrm{~nm}$, strip spacing is $1 \mu \mathrm{m}$, and length is $0.3-100 \mu \mathrm{m}$; aspect ratios are 1:1, 1:2, 1:3, 1:5, 1:10, and 1:333. The dynamic behavior was studied by network analyzer ferromagnetic resonance (FMR) using Permalloy strips on a coplanar waveguide in flip-chip geometry. The FMR mode frequencies $\left(f_{r}\right)$ can be controlled by the aspect ratio as well as by the applied magnetic field $(H)$. In longer strips (1:10 and 1:333), the excitation frequencies show a soft mode behavior $\left(H_{\text {eff }}=990\right.$ Oe $)$ when the field is along the hard axis. However, along the easy axis (along the strip length), $f_{r}$ increases with applied field. At a field of $3 \mathrm{kOe}, f_{r}$ values are almost independent of aspect ratio along the easy axis except for the 1:1 strip. Along the hard axis, the frequencies are strongly dependent upon the aspect ratio. We also observed that the frequency linewidths of the strips are dependent on the aspect ratio. (C) 2008 American Institute of Physics. [DOI: 10.1063/1.2839625]
\end{abstract}

\section{INTRODUCTION}

The continuous increase of storage density makes magnetic strip arrays very attractive for high density magnetic storage. The effect of going from continuous to patterned media on the various system components is generally benign. The principal advantage ${ }^{1,2}$ of patterned media is that they offer the possibility of overcoming the problem of the superparamagnetic limit on grain sizes. The high frequency dynamics of nanosized particles or strips are critically important for the understanding ${ }^{1-4}$ and improvement of their magnetization reversal characteristics. The finite lateral dimensions of magnetic strips changes the demagnetizing field affecting magnetic properties, especially the dynamical magnetic properties. In a real device, the time scale of the magnetization reversal process, studied mainly by ferromagnetic resonance (FMR) and Brillouin light scattering (BLS), in "in the nanosecond range." Recently, the study of spin waves ${ }^{1-4}$ in arrays of Permalloy (Py) nanoparticles has given evidence of frequency quantization of spin wave modes. Analytical models have been recently proposed to calculate the spin wave spectrum in circular and rectangular dots. ${ }^{5-7}$ FMR has an edge over BLS in the precise determination of the mode positions and linewidth.

In this study, we performed high frequency dynamic studies on Permalloy nanosized arrays of different aspect ratios (width to length) by using network analyzer FMR. The measurements were performed in the frequency domain, in contrast to the usual field swept measurements of standard FMR.

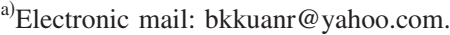

\section{EXPERIMENT}

A Permalloy film with nominal composition of $81 \% \mathrm{Ni}$ and $19 \% \mathrm{Fe}$ and thickness of $100 \mathrm{~nm}$ was deposited ${ }^{8}$ onto a resin by magnetron sputtering at the rate of $0.2 \mathrm{~nm} / \mathrm{s}$ at an Ar pressure of 3 mTorr. Fabrication of the arrays was performed using the method of electron beam nanolithography. ${ }^{8}$ Each patch had the form of an array of nanosized strips with a width of $300 \mathrm{~nm}$ separated by a distance of $1 \mu \mathrm{m}$. Final structures were made by a lift-off process, consisting in the removal of the resin and the overlaying film in an ultrasonic bath of acetone. A series of six samples consisting of strips with lengths of $300 \mathrm{~nm}, 600 \mathrm{~nm}, 900 \mathrm{~nm}, 1.5 \mu \mathrm{m}, 3 \mu \mathrm{m}$, and $100 \mu \mathrm{m}$ were fabricated, corresponding to the aspect ratios of the width to length given by 1:1, 1:2, 1:3, 1:5, 1:10, and $1: 333$.

In our earlier study, ${ }^{8}$ the quality of each pattern was tested using a field emission scanning electron microscope and an atomic force microscope (AFM). Magnetic hysteresis loops were measured at room temperature using a superconducting quantum interference device and microwave absorption was studied at room temperature by means of an X-band Bruker electron paramagnetic resonance spectrometer.

In the present work, a Cu-coplanar waveguide structure on a GaAs substrate was used as a transmission line to propagate an electromagnetic wave from a network analyzer. The nanostrip array was flipped on top of the transmission line with the strip length or width parallel to an external dc magnetic field. The frequency was swept from 0.05 to $40 \mathrm{GHz}$ at zero or a fixed external magnetic field $(H)$. The device characterization was done using a vector network analyzer along with a microprobe station. Noise, delay due to uncompensated transmission lines connectors, 

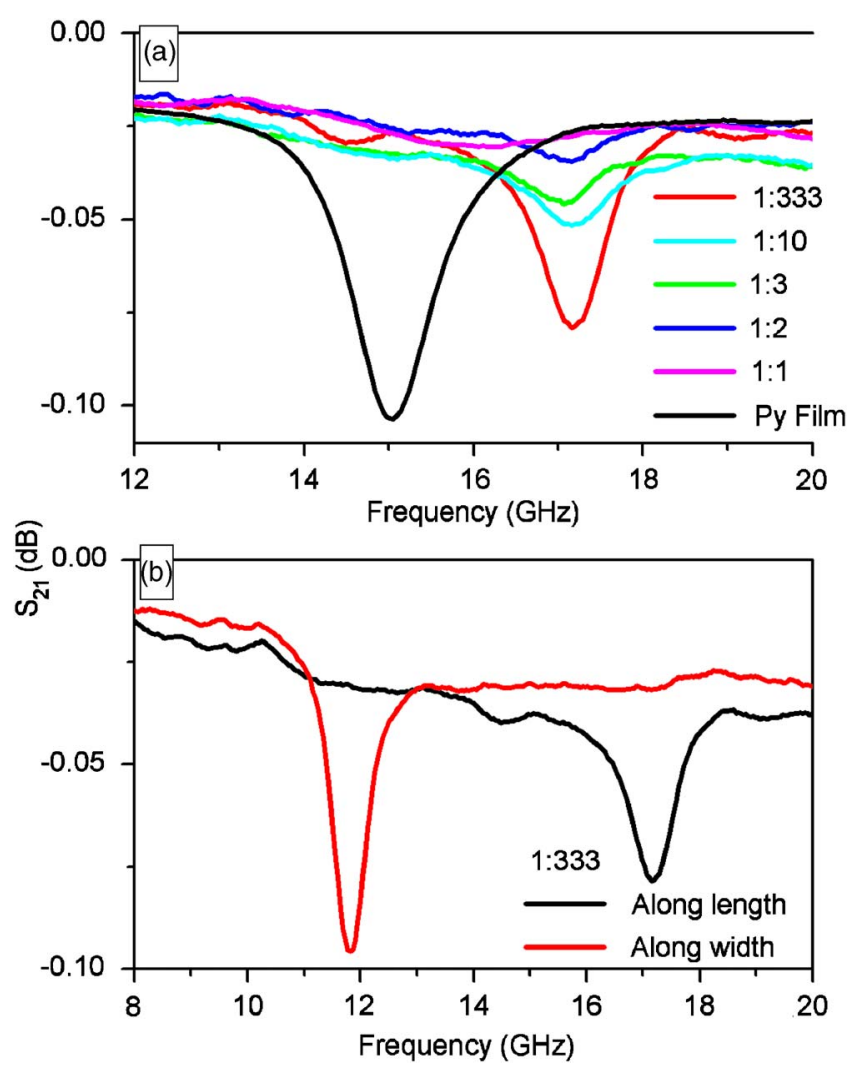

FIG. 1. (Color online) Part A shows the transmission response measured by the network analyzer of different Permalloy strips at a 3 kOe field applied along the length of the strips. The notch shows the resonance frequency of the strip. Part B shows the transmission response of a strip structure with aspect ratio 1:333 with the magnetic field applied along the length as well as the width of the strip, showing different resonance frequencies.

its frequency dependence, and cross-talk which occurred in measurement data, have been taken into account by performing through-open-line calibration using NIST MULTICAL ${ }^{\circledR}$ software. ${ }^{9}$ The results presented also include a background subtraction - the coplanar waveguide without the sample was measured and this was subtracted from the data with the sample. The width of the signal lines was $12 \mu \mathrm{m}$ and the length of the device was $6 \mathrm{~mm}$. The filters were designed for a $50 \Omega$ characteristic impedance. The exact resonance frequency $\left(f_{\text {res }}\right)$ and frequency linewidth $(\Delta f)$ were obtained from the Lorentzian fits to the experimental data.

\section{RESULTS AND DISCUSSION}

Network analyzer FMR (NA-FMR) spectra $\left(S_{21}\right.$ versus frequency) for Permalloy strips and a continuous film at a 3 kOe applied magnetic field are shown in Fig. 1 (part A). In comparison to the Py film $\left(f_{r}=15 \mathrm{GHz}\right.$ at $\left.3 \mathrm{kOe}\right)$, the spectra for the Py strips with the field along the long axis are shifted upward by $\sim 1,2,2.15,2.2$, and $2.5 \mathrm{GHz}$ for the $1: 1,1: 2$, $1: 3,1: 5$, and 1:10 strips, respectively.

Here, the microwave response of the array primarily reflects the properties of the individual strips because of the large spacing $(1 \mu \mathrm{m})$ between the nanosized strips and the $q=0$ wave vector. The upward shift of resonance frequency for longer strips is due to the increase in shape anisotropy with increase of length. In a microwave experiment, when

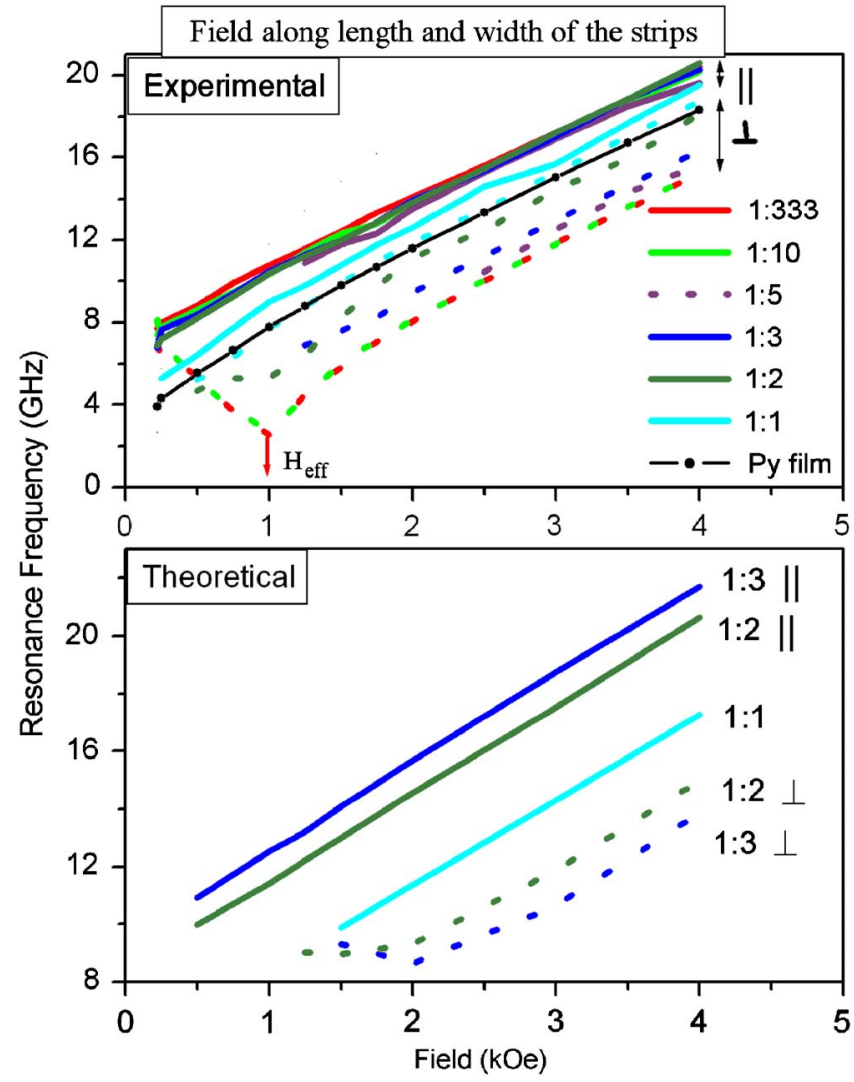

FIG. 2. (Color online) Resonance frequency vs applied magnetic field along the length and the width of the strip for structures with different aspect ratios and for a continuous Py film. The theoretical results from micromagnetics calculations are shown in the lower panel. For the simulations $M_{s}$ $=0.68 \mathrm{kG}$. The parallel and perpendicular signs indicate the direction of the applied field with respect to the length of the strip.

the magnetic field is applied along the length of the strip, the spins precess in the uniform mode at FMR and they generate a demagnetizing field with its origin in the "magnetic charges" present on the surfaces. These self-demagnetizing fields are responsible for the upshifting of the resonance frequency of the strip in comparison ${ }^{5}$ to a continuous film. The absorption spectra of all the strips show comparable line shape.

We note that experimentally we also found different frequencies for the 1:1 strip structure depending on the orientation of the magnetic field. It is not entirely clear what the origin of this effect is. The AFM of the array seems to indicate that the 1:1 structure is not quite square but is slightly elongated in one direction. In addition, the interactions among the different nano-objects may shift the resonances slightly.

Part B of Fig. 1 shows different resonance positions for 1:333 strip along the length $\left(f_{r}=11.8 \mathrm{GHz}\right)$ and the width $\left(f_{r}=17.2 \mathrm{GHz}\right)$ of the strip at an applied field of $3 \mathrm{kOe}$. This behavior of the resonance frequency is consistent with the presence of the uniaxial shape anisotropy of the strips and indicates that the shape anisotropy can cause significant changes in the frequency. The magnitude of this anisotropy is reflected in the difference in resonance frequencies between the cases where the field is along the length or the width of the strip.

Figure 2 shows the ensemble of the NA-FMR resonance 
frequencies of the Py film and Py strips with a magnetic field applied along the length and the width of the strips. As usual, the resonance frequency can be tuned by an external magnetic field. The resonance frequency generally increases with the increase of the applied field both when the field is applied along the length and the width of the strip for all the strips. However, for the 1:10 and 1:333 strips, there are two frequency-field regimes when the field is applied along the width of the strip. Here, the resonance frequency decreases linearly with a decrease of the magnetic field down to a certain value and then starts increasing with a decrease of field. This point of deviation of frequency-field data corresponds to the sample leaving its saturation state. The transition between the two regimes indicates the value of shape anisotropy. We derived the saturation magnetization, $g$ factor, and in-plane anisotropy of Py from a best fit procedure of the frequency-field data of the continuous Py film. The value of $M_{s}$ is relatively low at $0.612 \mathrm{kG}$. These results are used along with the self-demagnetization fields to calculate the shape anisotropy in strips of different lengths.

We also performed theoretical dynamical micromagnetic calculations for the uniform mode in different structures. We break the structure into small cells and use the LandauLifshitz equation,

$$
\frac{\partial \mathbf{M}}{\partial t}=-\frac{|\gamma|}{1+\alpha^{2}}\left[\mathbf{M} \times \mathbf{H}_{\mathrm{eff}}-\frac{\alpha}{M} \mathbf{M} \times\left(\mathbf{M} \times \mathbf{H}_{\mathrm{eff}}\right)\right],
$$

to calculate the motion of the magnetization within an individual cell. The effective field $\left(H_{\text {eff }}\right)$ acting on a cell is given by a sum of the dipole fields, effective exchange fields, and the applied field. Here, $\alpha$ is a dimensionless damping constant. To find the normal modes we used $M=.68 \mathrm{kG}, A$ $=1.3 \times 10^{-6} \mathrm{erg} / \mathrm{cm}$, and $\alpha=.0001$. The individual cell size was $100 \mathrm{~nm}$ in thickness and $10 \times 10 \mathrm{~nm}^{2}$ in the plane of the sample. A larger cell size was used for calculations of the larger structures. The calculations are similar to those carried out in Ref. 10, except that the demagnetizing terms are calculated using the Newell tensor. ${ }^{11}$ The results are presented in Fig. 2(b). The general trends seen in the experiment are also found in the theoretical results; however, there are some differences. As mentioned earlier the 1:1 structure shows two different frequencies (about $1 \mathrm{GHz}$ apart) depending on the orientation of the magnetic field. This is larger than the experimental uncertainty of about of $\pm 0.2 \mathrm{GHz}$. No such difference was found in the theory. Also, the soft modelike behavior described in the previous paragraph can be identified through the theoretical calculations as the sample undergoing a phase transition which is nucleated at the edges of the sample which are perpendicular to the applied field. The soft mode behavior seen experimentally in the longer structures is also found in the numerical calculations and is more pronounced than that for the shorter structures.

The frequency linewidth $\left(\Delta f_{r}\right)$ obtained from the NAFMR absorption spectra of the strips represents the signal from the whole sample. The frequency linewidth of the strips decreases as the length of the strip increases (Fig. 3) and is a minimum for a continuous film of the same thickness (separate star; $\Delta f_{r}=1.12 \mathrm{GHz}$ at $3 \mathrm{kOe}$ ). This reduction of the linewidth is due to the shape anisotropy. ${ }^{12}$ Figure 3 also

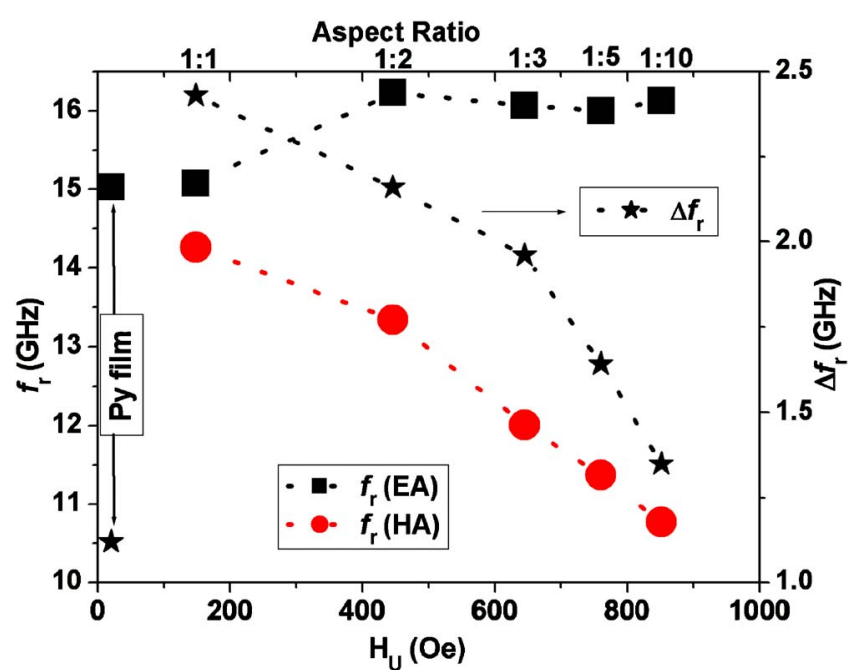

FIG. 3. (Color online) The resonance frequencies, (easy axis and hard axis) measured by NA-FMR and linewidth as a function of shape anisotropy $\left(H_{U}\right)$ at a magnetic field of $3 \mathrm{kOe}$. The top of the $X$ axis shows the aspect ratios of the strips $(1: 1,1: 2,1: 3,1: 5$, and 1:10).

shows data at a $3 \mathrm{kOe}$ field of resonance frequencies along the length and width of the strip and frequency linewidth as a function of shape anisotropy $\left(H_{u}\right)$.

\section{CONCLUSIONS}

Gyromagnetically induced resonance frequencies observed from NA-FMR along the length and the width of the Py strips are different and are a measure of shape anisotropy. The mode frequencies are observed to be controlled by the aspect ratio as well as by the applied magnetic field. For longer strips (1:10 and 1:333), there is a soft mode behavior when the magnetic field is applied along the strip width. We also observed that the frequency linewidths of the strips are dependent on the aspect ratio. The change in frequency of the uniform mode, observed in the patterned sample with respect to the continuous film, is primarily due to the selfdemagnetizing field inside the strips.

\section{ACKNOWLEDGMENTS}

The work at UCCS was supported by DOA under Grant No. W911NF-04-1-0247.

${ }^{1}$ S. O. Demokritov, B. Hillebrands, and A. N. Slavin, Phys. Rep. 348, 441 (2001).

${ }^{2}$ G. Carlotti and G. Gubbiotti, J. Phys. C 14, 8199 (2002).

${ }^{3}$ G. Gubbiotti, G. Carlotti, F. Nizzoli, R. Zivieri, T. Okuno, and T. Shinjo, IEEE Trans. Magn. 38, 2532 (2002).

${ }^{4}$ S. Demokritov and B. Hillebrands, Spin Dynamics in Confined Magnetic Structures I. Topics Appl. Phys., edited by B. Hillebrands and K. Ounadjela (Springer-Verlag, Berlin, 2002) pp. 65-92.

${ }^{5}$ R. Arias and D. Mills, Phys. Rev. B 63, 134439 (2001).

${ }^{6}$ K. Y. Guslienko and A. N. Slavin, J. Magn. Magn. Mater. 215, 576 (2000).

${ }^{7}$ R. W. Damon and J. R. Eshbach, J. Phys. Chem. Solids 19, 308 (1961).

${ }^{8}$ L. M. Malkinski, M. Yu, A. Y. Vovk, D. J. Scherer II, L. Spinu, W. Zhou, S. Whittenburg, and Z. Davis, and J.-S. Jung, J. Appl. Phys. 101, 09J110 (2007).

${ }^{9}$ R. B. Marks, IEEE Trans. Microwave Theory Tech. 39, 1205 (1991).

${ }^{10}$ M. Grimsditch, G. K. Leaf, H. G. Kaper, D. A. Karpeev, and R. E. Camley, Phys. Rev. B 69, 174428 (2004).

${ }^{11}$ A. J. Newell, J. Geophys. Res. 98, 9551 (1993).

${ }^{12}$ B. Kuanr, R. E. Camley, and Z. Celinski, Appl. Phys. Lett. 87, 012502 (2005). 